\title{
Asymmetric Linkage Disequilibrium: Tools for Assessing Multiallelic LD
}

\author{
Richard M. Single ${ }^{1 *}$, Nick Strayer ${ }^{1}$, Glenys Thomson ${ }^{2}$, Vanja Paunic ${ }^{3}$, Mark Albrecht ${ }^{3}$, Martin \\ Maiers $^{3}$, \\ ${ }^{1}$ Department of Mathematics and Statistics, University of Vermont, Burlington, VT \\ ${ }^{2}$ University of California, Berkeley, California \\ ${ }^{3}$ National Marrow Donor Program, Minneapolis, Minnesota \\ *Corresponding Author: \\ richard.single@uvm.edu \\ Department of Mathematics and Statistics \\ University of Vermont \\ Burlington, VT 05405 \\ 802-656-8631 (Phone) \\ 802-656- 2552 (FAX)
}

\begin{abstract}
Standard measures of linkage disequilibrium (LD) provide an incomplete description of the correlation between two loci. Recently, Thomson and Single (2014) described a new asymmetric pair of LD measures (ALD) that give a more complete description of LD. The ALD measures are symmetric and equivalent to the correlation coefficient $r$ when both loci are bi-allelic. When the numbers of alleles at the two loci differ, the ALD measures capture this asymmetry and provide additional detail about the LD structure. In disease association studies the ALD measures are useful for identifying additional disease genes in a genetic region, by conditioning on known effects. In evolutionary genetic studies ALD measures provide insight into selection acting on individual amino acids of specific genes, or other loci in high LD (see Thomson and Single (2014) for these examples). Here we describe new software for computing and visualizing ALD. We demonstrate the utility of this software using haplotype frequency data from the National Marrow Donor Program (NMDP). This enhances our understanding of LD patterns in the NMDP data by quantifying the degree to which LD is asymmetric and also quantifies this effect for individual alleles.
\end{abstract}

\section{Introduction}

Linkage disequilibrium (LD) is the non-random association of alleles at two or more loci.

Understanding and incorporating the LD structure of a genetic region into analyses is necessary for detecting disease predisposing variants, as well as for understanding the evolutionary history of a genetic region.

The basic unit of LD is defined as $D_{i j}=f_{i j}-p_{A i} p_{B j}$, where $f_{i j}$ is the frequency for the $A_{i} B_{j}$ haplotype and $p_{A i}$ and $p_{B j}$ are frequencies for alleles $A_{i}$ and $B_{j}$, respectively. Since the maximum value that $D_{i j}$ can assume is a function of the observed allele frequencies, there are several normalized measures of the strength of LD for bi- and multi-allelic data $[1,2]$. The two most common measures of the strength of LD are: (1) the normalized measure of the individual LD values [3], $D_{i j}{ }^{\prime}=D_{i j} / D_{\max }$, and (2) the correlation coefficient $r$ for bi-allelic data, often reported as $r^{2}=D_{i j}{ }^{2} /\left(p_{A 1} p_{A 2} p_{B 1} p_{B 2}\right)$. 
The ALD measures [4] can be written as normalized versions of an overall allele specific homozygosity statistic. The ordinary homozygosity $(F)$ statistic, $F_{A}=\Sigma_{i} p_{A i}{ }^{2}$, extends to a conditional two locus statistic, called Haplotype or Allele Specific Homozygosity (ASF). It measures the level of genetic variation at locus $A$ on haplotypes with the $B_{j}$ allele at locus $B$ (and vice versa), $F_{A / B j}=\Sigma_{i}\left(f_{i j} /\right.$ $\left.p_{B j}\right)^{2}$ [5]. When $A S F=1.0$ there is complete correlation of the genetic variation at the two loci on the specified conditional haplotype. Lower values of ASF indicate a relatively larger amount of variation at the other locus on the conditional haplotype. A weighted average of ASF values, over all alleles at the conditioned upon locus, gives the overall measure of ASF, $F_{A / B}=\Sigma_{j}\left(F_{A / B j}\right)\left(p_{B j}\right)$. A similar result, swapping the roles of locus $\mathrm{A}$ and $\mathrm{B}$ in the above formula, is obtained by conditioning on the $\mathrm{A}$ locus.

The squared ALD statistic, conditioning on locus B, is a standardized measure of the difference between these conditional and unconditional homozygosity measures: $W_{A / B}{ }^{2}=\left(F_{A / B}-F_{A}\right) /\left(1-F_{A}\right)$. Swapping the roles of the A and B subscripts gives the measure conditioned on variation at the $A$ locus. When the relationship is symmetric, the squared ALD measures are equal to $W_{n}^{2}$, the multiallelic extension of the $r^{2}$ measure also known as Cramer's V statistic[6]. In the bi-allelic case they collapse to the well-known $r^{2}$ measure.

\section{Methods - Asymmetric LD Web Application}

Using the R shiny server [7] we developed a web application that computes the ALD measures and visualizations of the measures and their components.

The ALD app allows the user to upload data in two formats. The first format is a file of multilocus haplotypes for any number of loci followed by the haplotype frequency. The first row must be a header with the locus names and an arbitrary name for the column of frequencies. There are $k+1$ columns, where $k$ is the number of loci (Table 1). The second file format has a list of pairwise haplotype frequencies. There are five columns that must have the names locus1, locus2, allele1, allele2, and haplo.freq. The order of the loci in the input file determines the order for plotting ALD statistics.

For a set of haplotypes, the sum of the frequencies should be equal to one, but there are situations where the sum may be less than one (e.g., when very low frequency haplotypes are not reported). In order to accommodate this situation, the ALD app has a "tolerance" parameter that can be set by the user (Figure 1). In order for the app to begin computations, the sum of the haplotype frequencies must be within plus or minus tolerance of 1.0. The default value for the tolerance is 0.01 .

The combination of ASF and allele frequency directly indicates the extent to which specific haplotypes contribute to the overall extent of asymmetric LD. Because of this, we decided to conditionally format the entries in the ASF table to visually highlight those alleles that have large contributions to overall $\mathrm{LD}$ when conditioning on one of the loci. This was accomplished using the $\mathrm{d} 3$.js javascript library to scale colors from green to red based on a linear scale from zero to the observed maximum for haplotype frequencies and zero to one for the ASF values. Scaling is done separately for the ASF data and allele frequency data.

\section{Results - Application to NMDP Haplotype Frequency data}

We applied the ALD measures to data for high resolution HLA haplotypes in the US population [8] downloaded from the NMDP since this is the largest publically available dataset of HLA haplotype 
frequencies used for clinical and research applications. The data consist of haplotype frequency estimates for HLA-A, -C, -B, -DRB1, and -DQB1 from 21 detailed populations and five broad U.S. Census categories of race and ethnicity. In what follows we describe results from the European American/Caucasian (abbr: CAU) and Hispanic (abbr: HIS) broad race/ethnicity populations.

Figures 1 and 2 have ALD plots for the CAU and HIS broad groups, respectively. The figures show a good deal of asymmetry in the LD relationships for the $\mathrm{C} \sim \mathrm{B}$ and the DRB1 DQB1 locus pairs. For the CAU population, the overall variation for DQB1 is relatively low given specific DRB1 alleles $\left(W_{D Q B 1 \mid D R B 1}=0.92\right)$ while overall variation at DRB1 is greater given specific DQB1 alleles $\left(W_{D R B 1 \mid D Q B 1}=0.74\right)$. A similar, but even more extreme degree of asymmetry is seen in the HIS population.

Figures 3 and 4 have allele specific homozygosity (ASF) values for a set of DRB1 DQB1 pairs. Figure 3 also contains the allele frequency for specific alleles (focal alleles) at each locus. The combination of these values provides insight into the asymmetric pattern of LD seen for this pair of loci. For example, in the HIS population the ASF value is 1.0 for $\mathrm{DRB} 1 * 01: 02$, indicating that there is no variability at the DQB1 locus for haplotypes containing DRB1*01:02. However, the contribution to overall ALD by this haplotype is moderated by the lower allele frequency for the focal allele in the HIS population and even lower frequency in the CAU population. The DRB $1 * 03: 01$ allele has a relatively high frequency in both population along with a high ASF indicating that it contributes substantially to ALD in each population.

The highlighting/coloring of the ASF and allele frequency values allows one to easily see combinations that contribute to high LD. The higher $W_{D Q B 1 \mid D R B 1}$ values for the CAU and HIS populations are explained by the larger fraction of red-orange and orange-orange pairings (high ASF and high allele frequency) in the CAU and HIS populations. The lower $W_{D R B 1 \mid D Q B 1}$ value in the HIS population compared to the CAU population is highlighted by the lack of pairings in the red-orange end of the spectrum for the HIS population compared to the CAU.

From Figure 3 it is clear that the DQB1*06:02 allele makes a large contribution to $W_{D R B 1 \mid D Q B 1}$ in the CAU population, since it has high ASF and allele frequency values. DQB1*06:02 has an allele frequency of 0.1287 and ASF value of 0.9906. The DRB1*15:01 DQB $1 * 06: 02$ haplotype accounts for $99.5 \%$ of the DRB1 DQB1 haplotype frequencies where DQB1*06:02 is the focal allele. (see supplementary Table 1). Thus, although there are a large number of DRB1 alleles (24 different ones) that occur with DQB1*06:02 in haplotypes, their frequency contribution is very small.

In the HIS population, there is no DQB1 allele with frequency greater than 0.05 and ASF value greater than 0.70 . Figure 3 shows that DQB1*06:02 is relatively common with a frequency of 0.0759 , but an ASF value of only 0.6825 . While the DRB1*15:01 DQB1*06:02 haplotype is the most frequent DRB1 DQB1 haplotype with DQB1*06:02 as the focal allele, it only accounts for $81.2 \%$ of the DRB1 DQB1 haplotype frequency where DQB1*06:02 is the focal allele (see supplementary Table 2).

\section{Discussion}

Since the input to the ALD app is haplotype frequency data, it can be used with data from a variety of public data repositories. There is a large literature on the accuracy of haplotype frequency estimation (see [9] for recent references). Issues of estimation accuracy are particularly relevant for highly polymorphic data and the accuracy of LD estimates will be affected by that of the haplotype frequency data used to compute them. 
ALD is a valuable tool for Hematopoietic Stem Cell (HSC) registries because it can inform optimal typing strategies. The Bone Marrow Donors Worldwide (BMDW.org) database lists HLA typing information on over 25 Million volunteer donors and cord blood units. Although the clinical standard for matching is currently to match at 10/10 alleles (5 HLA loci with 2 alleles each) the majority $(54.6 \%)$ of donors in the database lack HLA-C typings and an even larger proportion lack HLA-DQB1 typings $(72.6 \%)$.

Based on the ALD results (Figures 3 and 4) these are the two least informative loci since the ALD values are $0.87 \& 0.85$ for HLA-C conditional on HLA-B and 0.92 and 0.94 for HLA-DQB1 conditional on HLA-DRB1 in two representative populations. As clinical standards evolve to include more loci (e.g. HLA-DPB1, HLA-DQA1, MHC SNPs) the ALD measure will be a useful tool for informing decisions about how many donors to retype at these loci and how much information one can expect to glean from this additional typing beyond that which can be recovered through imputationbased matching algorithms [10].

\section{Acknowledgments}

This work was supported in part by a REACH grant from the University of Vermont and the Office of Naval Research (ONR) grant N00014-14-1-0848. The content is solely the responsibility of the authors and does not necessarily represent the official views of the ONR. The authors would like to thank Jordan Bakerman for initial feedback on the software. RS and GT developed the methodology. RS developed the software framework. RS, NS, VP, and MA developed the web application. RS and MM wrote the manuscript. NS, GT, VP, and MA reviewed the manuscript. 


\begin{tabular}{llllll} 
A & C & B & DRB1 & DQB1 & freq \\
\hline 01:01g & $01: 02$ & $15: 01 \mathrm{~g}$ & $01: 01$ & $05: 01$ & 0.00006 \\
01:01g & $01: 02$ & $15: 01 \mathrm{~g}$ & $09: 01$ & $03: 03$ & 0.00006 \\
01:01g & $01: 02$ & $15: 01 \mathrm{~g}$ & $13: 01$ & $06: 03$ & 0.00007 \\
01:01g & $01: 02$ & $27: 05 \mathrm{~g}$ & $01: 01$ & $05: 01$ & 0.00016 \\
$\ldots$ & & & & &
\end{tabular}

Table 1: multilocus haplotype frequency file format

\begin{tabular}{lllll} 
locus1 & locus2 & allele1 & allele2 & haplo.freq \\
\hline A & C & $01: 01$ & $07: 01$ & 0.07435 \\
A & C & $02: 01$ & $03: 04$ & 0.05002 \\
$\ldots$ & & & & \\
DRB1 & DQB1 & $07: 01$ & $06: 02$ & 0.00168 \\
DRB1 & DQB1 & $11: 01$ & $03: 02$ & 0.0025
\end{tabular}

Table 2: pairwise haplotype frequency file format 
Figure 1: Screenshot of the ALD web app. Note that the tolerance value needed to be set to 0.02 since the sum of the haplotype frequencies was 0.98. The plot has ALD measures for Classical HLA Genes in the Caucasian (CAU) broad race category of the NMDP Donor population. Data Source: Six-locus high resolution HLA haplotype frequencies derived from mixed-resolution DNA typing for the entire US donor registry (Gragert et al. 2013).

Figure 2: ALD Measures for Classical HLA Genes in the Hispanic (HIS) broad race category of the NMDP Donor population. Data Source: as in Figure 1.

Figure 3: Allele specific homozygosity (ASF) and allele frequency values for certain DRB1 DQB1 pairs in the Caucasian broad race category (CAU). The focal allele is the allele conditioned upon and the ASF value is the homozygosity value computed by conditioning on this allele. High ASF values for higher frequency alleles make a large contribution to overall ALD. Data Source: as in Figure 1.

Figure 4: Allele specific homozygosity (ASF) and allele frequency values for certain DRB1 DQB1 pairs in the Hispanic broad race category (HIS). Data Source: as in Figure 1.

\section{References}

[1] Hedrick PW: Gametic disequilibrium measures: proceed with caution. Genetics 1987;117:331.

[2] Lewontin RC: On measures of gametic disequilibrium. Genetics 1988;120:849.

[3] Lewontin RC: The Interaction of Selection and Linkage. I. General Considerations; Heterotic Models. Genetics 1964;49:49.

[4] Thomson G, Single RM: Conditional asymmetric linkage disequilibrium (ALD): extending the biallelic r2 measure. Genetics 2014;198:321.

[5] Malkki M, Single R, Carrington M, Thomson G, Petersdorf E: MHC microsatellite diversity and linkage disequilibrium among common HLA-A, HLA-B, DRB1 haplotypes: implications for unrelated donor hematopoietic transplantation and disease association studies. Tissue Antigens 2005;66:114.

[6] Cramer H: Mathematical Methods of Statistics. Princeton: Princeton University Press; 1946.

[7] Chang W, Cheng J., Allaire, J., Xie, Y., McPherson: shiny: Web Application Framework for R. 2015. R package version 0.11.1, http://shiny.rstudio.com.

[8] Gragert L, Madbouly A, Freeman J, Maiers M: Six-locus high resolution HLA haplotype frequencies derived from mixed-resolution DNA typing for the entire US donor registry. Hum Immunol 2013;74:1313.

[9] Pappas DJ, Tomich A, Garnier F, Marry E, Gourraud PA: Comparison of high-resolution human leukocyte antigen haplotype frequencies in different ethnic groups: Consequences of sampling fluctuation and haplotype frequency distribution tail truncation. Hum Immunol 2015;76:374.

[10] Madbouly A, Gragert L, Freeman J, Leahy N, Gourraud PA, Hollenbach JAet al. : Validation of statistical imputation of allele-level multilocus phased genotypes from ambiguous HLA assignments. Tissue Antigens 2014;84:285. 
Figure 1

Asymmetric LD (ALD) Upload Data ALD Table Allele Specific Homozygosity Raw Data Ctation

Upload a Haplotype Frequency File

Choose Fle ...B1_BroadRace-CAU.cSV Upload complete

Cick here to see the fle formats Separator
- Comma
Semicolon
(1) Tab

tolerance (for sum of haplo.freqs)

0.02

ALD plot options

$\checkmark$ Show ALD values

\section{\begin{tabular}{|l|l|l|}
\hline & $A$ & $L$ \\
\hline & & $D$ \\
\hline
\end{tabular} \\ Asymmetric Linkage Disequilibrium $L \quad D$}

NOTE: the sum of hapltoype frequencies needs to be within $+/-$ tolerance of 1.0 .

If the sum is not within this range, you must increase the tolerance value.

Sum of haplo.freqs $=0.988118089$

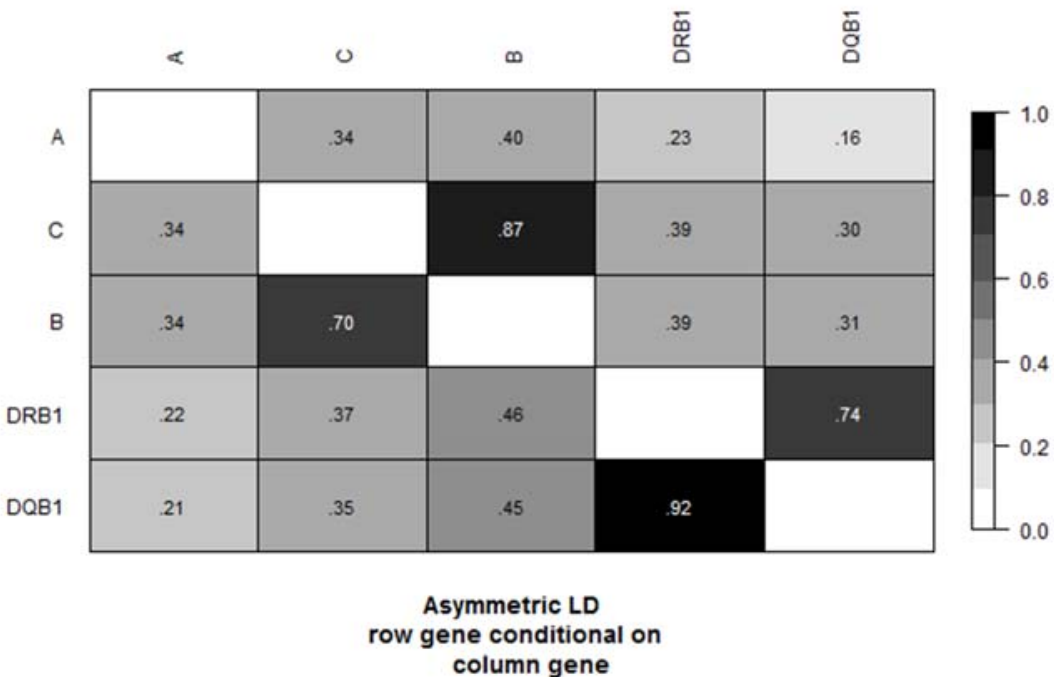


Figure 2

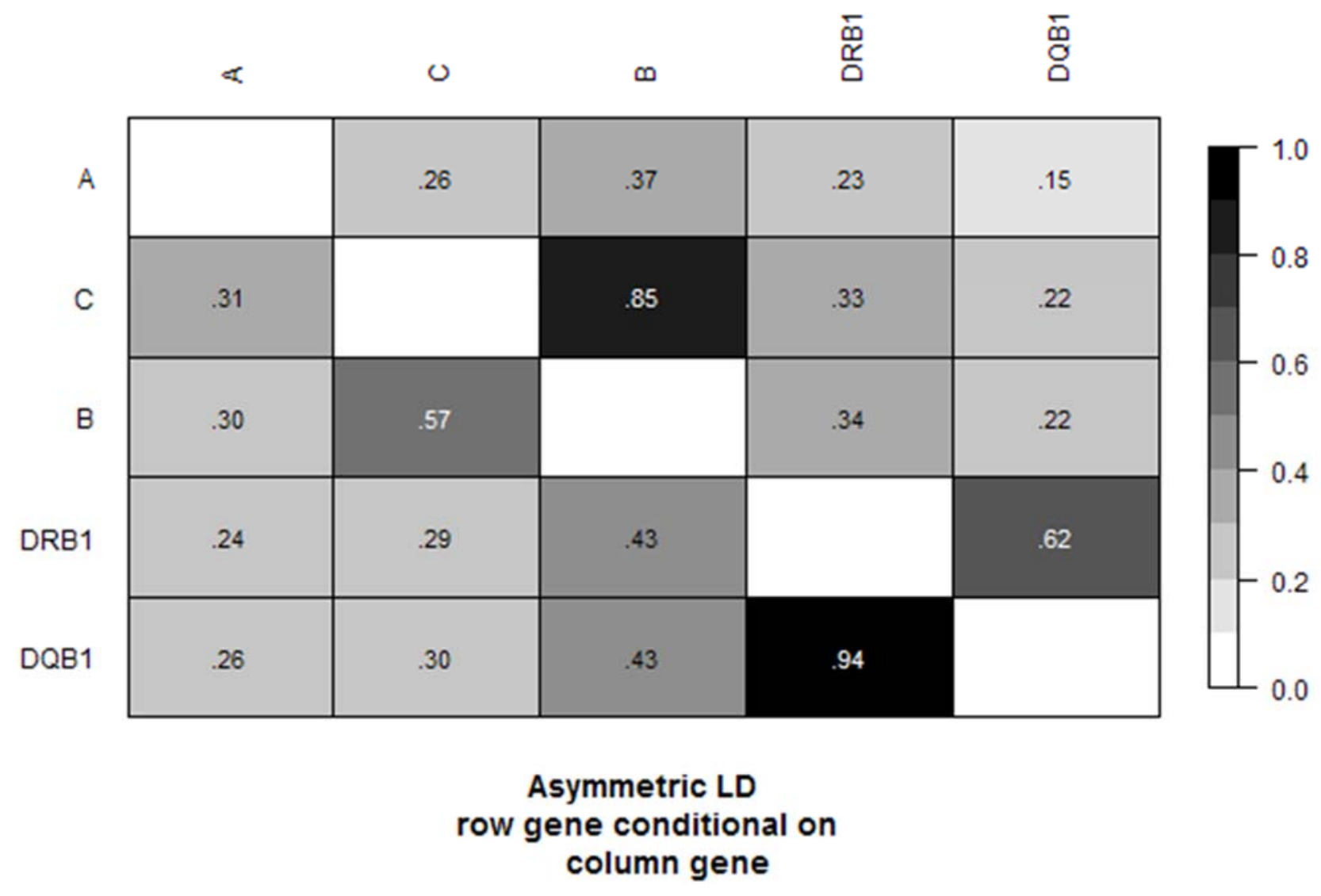


Figure 3a

ALD Table Allele Specific Homozygosity Raw Data Citation

Show $25 \quad$ entries $\quad$ Search:

\begin{tabular}{|c|c|c|c|c|}
\hline loci & focal & allele & allele.freq & as.homz \\
\hline DRB1-DQB1 & DRB1 & DRB1*07:01 & 0.130515064 & 0.6160090 \\
\hline DRB1-DQB1 & DRB1 & DRB1*15:01 & 0.130195915 & 0.9685337 \\
\hline DRB1-DQB1 & DRB1 & DRB1*03:01 & 0.114896983 & 0.9984061 \\
\hline DRB1-DQB1 & DRB1 & DRB1*01:01 & 0.085754340 & 0.9890753 \\
\hline DRB1-DQB1 & DRB1 & DRB1*04:01 & 0.080899916 & 0.5088757 \\
\hline DRB1-DQB1 & DRB1 & DRB1*11:01g & 0.063742614 & 0.9964338 \\
\hline DRB1-DQB1 & DRB1 & DRB1*13:01 & 0.062135957 & 0.9971113 \\
\hline DRB1-DQB1 & DRB1 & DRB1*13:02 & 0.046375042 & 0.6925585 \\
\hline DRB1-DQB1 & DRB1 & DRB1*11:04 & 0.036406796 & 0.9498116 \\
\hline DRB1-DQB1 & DRB1 & DRB1*04:04 & 0.034139333 & 0.9274368 \\
\hline DRB1-DQB1 & DRB1 & DRB1*14:01g & 0.027103872 & 0.9961778 \\
\hline DRB1-DQB1 & DRB1 & DRB1*08:01 & 0.023698311 & 0.9901462 \\
\hline DRB1-DQB1 & DRB1 & DRB1*16:01 & 0.017360643 & 0.9996086 \\
\hline DRB1-DQB1 & DRB1 & DRB1*12:01g & 0.017355707 & 0.9983291 \\
\hline DRB1-DQB1 & DRB1 & DRB1*01:02 & 0.015078181 & 0.9999215 \\
\hline DRB1-DQB1 & DRB1 & DRB $1 * 04: 02$ & 0.012664294 & 0.9997942 \\
\hline DRB1-DQB1 & DRB1 & DRB1*13:03 & 0.011921988 & 0.9990803 \\
\hline DRB1-DQB1 & DRB1 & DRB1*04:07g & 0.009666515 & 0.9812778 \\
\hline DRB1-DQB1 & DRB1 & DRB1*09:01 & 0.009648458 & 0.9351685 \\
\hline
\end{tabular}




\section{Figure $3 b$}

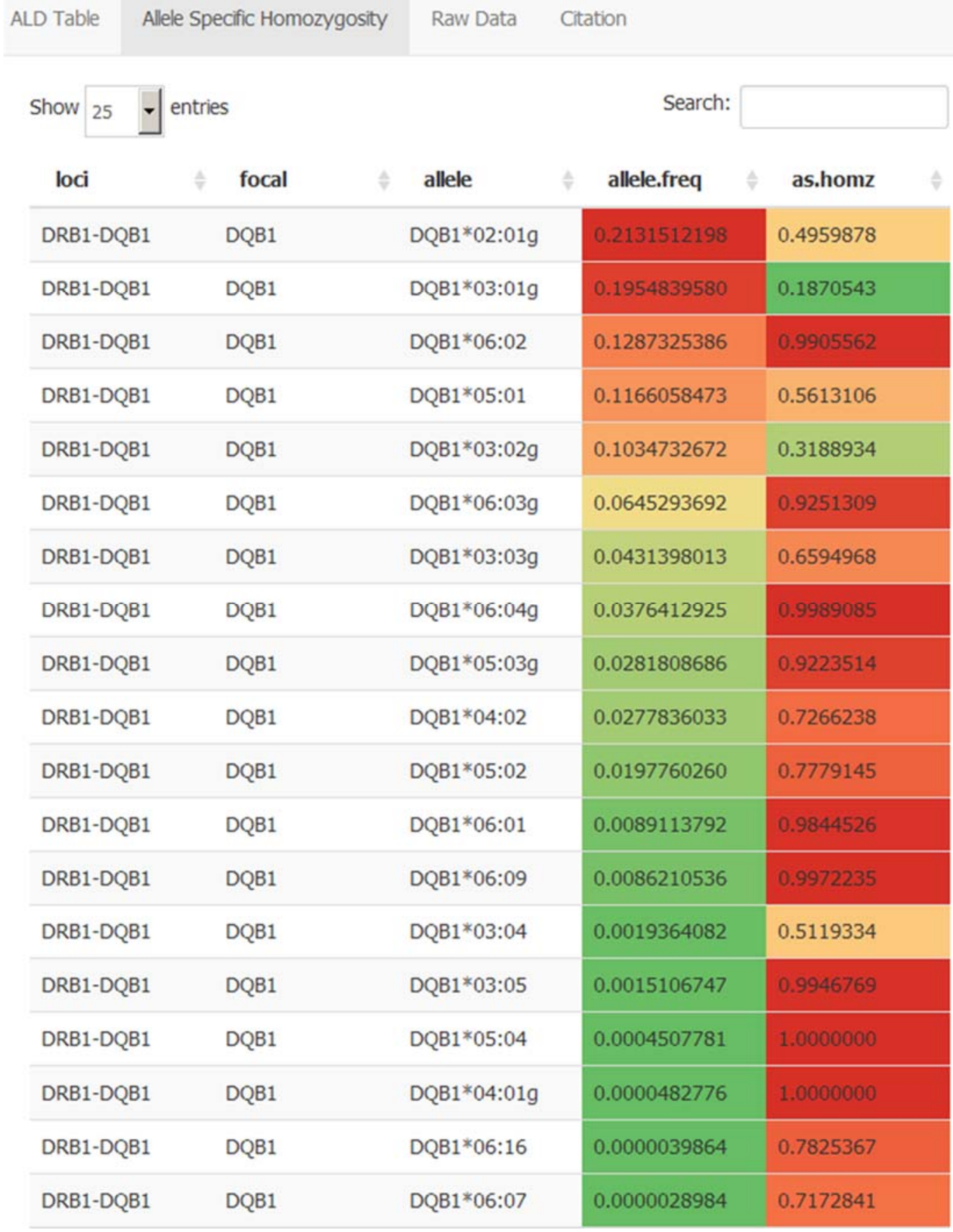


Figure 4a

ALD Table Allele Specific Homozygosity Raw Data Citation

Show $25 \quad$ entries

Search:

\begin{tabular}{|c|c|c|c|c|}
\hline loci & focal & allele & allele.freq & as.homz \\
\hline DRB1-DQB1 & DRB1 & DRB1*07:01 & 0.10966997 & 0.8084626 \\
\hline DRB1-DQB1 & DRB1 & DRB1*03:01 & 0.07145245 & 1.0000000 \\
\hline DRB1-DQB1 & DRB1 & DRB1*08:02 & 0.07055771 & 0.9945510 \\
\hline DRB1-DQB1 & DRB1 & DRB1*04:07g & 0.06617131 & 0.9601870 \\
\hline DRB1-DQB1 & DRB1 & DRB1*15:01 & 0.06235957 & 0.9762197 \\
\hline DRB1-DQB1 & DRB1 & DRB1*04:04 & 0.04736959 & 0.9953561 \\
\hline DRB1-DQB1 & DRB1 & DRB1*13:01 & 0.04573315 & 0.9818664 \\
\hline DRB1-DQB1 & DRB1 & DRB1*01:01 & 0.04572409 & 0.9987113 \\
\hline DRB1-DQB1 & DRB1 & DRB $1 * 11: 01 \mathrm{~g}$ & 0.04056052 & 0.8774626 \\
\hline DRB1-DQB1 & DRB1 & DRB1*13:02 & 0.04049713 & 0.5421140 \\
\hline DRB1-DQB1 & DRB1 & DRB $1 * 01: 02$ & 0.03500178 & 1.0000000 \\
\hline DRB1-DQB1 & DRB1 & DRB1*11:04 & 0.03057164 & 0.9386669 \\
\hline DRB1-DQB1 & DRB1 & DRB1*14:06 & 0.02843409 & 1.0000000 \\
\hline DRB1-DQB1 & DRB1 & DRB $1 * 04: 05$ & 0.02349153 & 0.7058320 \\
\hline DRB1-DQB1 & DRB1 & DRB1*16:02 & 0.02035360 & 0.8888536 \\
\hline DRB1-DQB1 & DRB1 & DRB1*04:11 & 0.02019289 & 0.7761968 \\
\hline DRB1-DQB1 & DRB1 & DRB1*14:02 & 0.01995422 & 0.8567913 \\
\hline DRB1-DQB1 & DRB1 & DRB1*04:01 & 0.01835482 & 0.5008228 \\
\hline DRB1-DQB1 & DRB1 & DRB1*14:01g & 0.01807481 & 0.9921390 \\
\hline
\end{tabular}




\section{Figure 4b}

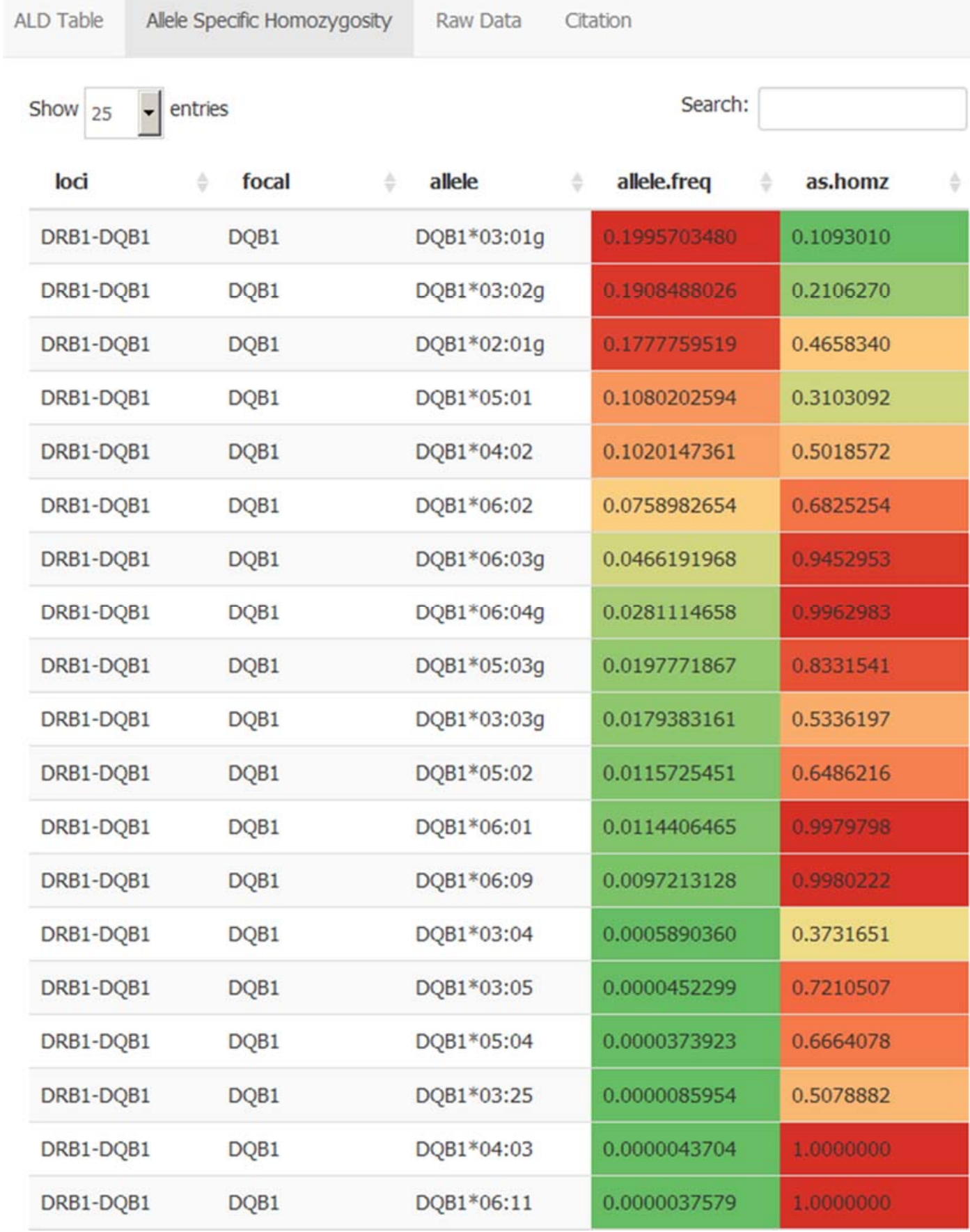

